\title{
Neutrino parameters from reactor and accelerator neutrino experiments
}

\author{
Manfred Lindner, Werner Rodejohann, and Xun-Jie Xu \\ Max-Planck-Institut für Kernphysik, Postfach 103980, D-69029 Heidelberg, Germany
}

(Received 17 January 2018; published 19 April 2018)

\begin{abstract}
We revisit correlations of neutrino oscillation parameters in reactor and long-baseline neutrino oscillation experiments. A framework based on an effective value of $\theta_{13}$ is presented, which can be used to analytically study the correlations and explain some questions including why and when $\delta_{C P}$ has the best fit value of $-\pi / 2$, why current and future long-baseline experiments will have less precision of $\delta_{C P}$ around $\pm \pi / 2$ than that around zero, etc. Recent hints on the $C P$ phase are then considered from the point of view that different reactor and long-baseline neutrino experiments provide currently different best-fit values of $\theta_{13}$ and $\theta_{23}$. We point out that the significance of the hints changes for the different available best-fit values.
\end{abstract}

DOI: 10.1103/PhysRevD.97.075024

\section{INTRODUCTION}

Short-baseline reactor and long-baseline accelerator neutrino experiments are of huge importance in the era of neutrino oscillation precision measurements. The precision determination of $\theta_{23}$, and the measurements of the $C P$ phase $\delta_{C P}$ and the neutrino mass ordering would help further understanding the leptonic flavor sector and could bring various insights in models behind neutrino mass and lepton mixing.

The interplay of long- and short-baseline experiments is exemplified by the dependence of the electron antineutrino survival probability in reactor experiments, which depends on $\theta_{13}$, and the $\nu_{\mu} \rightarrow \nu_{e}\left(\right.$ or $\bar{\nu}_{\mu} \rightarrow \bar{\nu}_{e}$ ) transition probability in accelerator experiments, which depends on $\theta_{13}, \theta_{23}, \delta_{C P}$ and the mass ordering. Inserting the reactor determination of $\theta_{13}$, as well as $\theta_{23}$ values from atmospheric data or longbaseline muon neutrino survival probabilities, into $\nu_{\mu} \rightarrow \nu_{e}$ (or $\bar{\nu}_{\mu} \rightarrow \bar{\nu}_{e}$ ) measurements can give hints on the $C P$ phase [1,2]. Combining $\nu_{\mu} \rightarrow \nu_{e}$ plus $\bar{\nu}_{\mu} \rightarrow \bar{\nu}_{e}$ data with additional input on $\theta_{23}$ is enough to get sensitivity on $\delta_{C P}[3,4]$. As matter effects start to play a non-negligible role also some sensitivity on the mass ordering can occur by combining different data sets. Indeed, recently hints towards nontrivial values of the $C P$ phase $\delta_{C P}$ and some preference of the normal ordering over the inverted one were found in combining short-baseline reactor and longbaseline accelerator neutrino experiments; see Refs. [5-7] for recent global fits.

Published by the American Physical Society under the terms of the Creative Commons Attribution 4.0 International license. Further distribution of this work must maintain attribution to the author(s) and the published article's title, journal citation, and DOI. Funded by SCOAP.
In this respect, one should note here the different values of $\theta_{13}$ that have been determined by the three reactor neutrino experiments: $\theta_{13}=8.43_{-0.17}^{\circ+0.17}$ (Daya Bay [8]), $8.62_{-0.57}^{\circ+0.54}$ (RENO [9]) and $9.73_{-0.85}^{\circ+0.79}$ (Double Chooz [10]). It is possible that the central value of $\theta_{13}$ is shifted (e.g. from a joint analysis of the collaborations), which will then have consequences on the determination of the other neutrino parameters. Moreover, the best-fit values (normal ordering for definiteness) of $\theta_{23}$ are $46.8^{\circ}$ from T2K [11] and $39.5^{\circ}\left(52.2^{\circ}\right)$ from $\mathrm{NO} \nu \mathrm{A}$ [12], where the latter has two almost equally good best-fit points. ${ }^{1}$ In this paper we try to estimate the possible impact of the different central values of $\theta_{13}$ and $\theta_{23}$ on the hints for $C P$ violation and the normal mass ordering. Towards this end, we reanalyze the correlation of long-baseline $\nu_{\mu} \rightarrow \nu_{e}\left(\bar{\nu}_{\mu} \rightarrow \bar{\nu}_{e}\right)$ transition and reactor $\bar{\nu}_{e} \rightarrow \bar{\nu}_{e}$ survival oscillations.

We study the impact of combining accelerator neutrino data with antineutrino data and with reactor data. We investigate the impact of the different $\theta_{13}$ and $\theta_{23}$ bestfit values on the determination of $\delta_{C P}$, as well as on the preference for the normal mass ordering. We demonstrate that the significance can shift and should thus be taken with care. We also remark that the theoretically very interesting ${ }^{2}$ value of $\delta_{C P}=-\pi / 2$ can be measured with currently running accelerator experiments better if future runs are not equally shared $1: 1$ in neutrino and antineutrino modes, but rather $2: 1$.

\footnotetext{
${ }^{1}$ Most recent data from $\mathrm{NO} \nu \mathrm{A}$ [13] does not seem to confirm this feature any longer. Changes of such best-fit points are typical for experiments with comparably low event numbers. For the sake of illustration of the general situation in the field, we will work with the two best-fit points from [12].

${ }^{2}$ See Refs. [14-36] for an incomplete list.
} 
The remainder of this paper is organized as follows. In Sec. II, we perform semianalytical analyses on the interplay of the neutrino parameters, including the impact of the different available central values of $\theta_{13}$ and $\theta_{23}$ on the $C P$ phase $\delta_{C P}$ and the mass ordering. In Sec. III, we further adopt numerical calculations to quantitatively study the phenomenology, which not only verifies our analyses in Sec. II but also gives a more precise description. Finally, we summarize in Sec. IV and leave some detailed issues concerning the semianalytical analyses in the appendix.

\section{ANALYTICAL APPROACH: EFFECTIVE $\theta_{13}$}

We start from the approximate neutrino oscillation formula [37-39] that can accurately describe $\nu_{\mu} \rightarrow \nu_{e}$ transitions in current and upcoming accelerator neutrino experiments such as T2K [1,40], MINOS [41], NO $\nu \mathrm{A}$ [42,43], DUNE [44] or T2HK [45]:

$$
\begin{aligned}
P\left(\nu_{\mu} \rightarrow \nu_{e}\right) \approx & 4 s_{13}^{2} c_{13}^{2} s_{23}^{2} \frac{\sin ^{2}(1-A) \Delta}{(1-A)^{2}} \\
& -8 \alpha J_{C P} \sin \Delta \frac{\sin A \Delta \sin (1-A) \Delta}{A} \frac{1-A}{\sin A \Delta} \frac{\sin (1-A) \Delta}{A} \\
& +8 \alpha\left(J_{C P} \cot \delta_{C P}\right) \cos \Delta \frac{\sin ^{2} A \Delta}{A^{2}} \\
& +4 \alpha^{2} s_{12}^{2} c_{12}^{2} c_{23}^{2}
\end{aligned}
$$

Here $\left(s_{i j}, c_{i j}\right) \equiv\left(\sin \theta_{i j}, \cos \theta_{i j}\right), \alpha \equiv \Delta m_{21}^{2} / \Delta m_{31}^{2}, \quad A \equiv$ $2 \sqrt{2} G_{F} N_{e} E / \Delta m_{32}^{2}\left(N_{e}\right.$ is the electron number density in matter), $\Delta \equiv \Delta m_{32}^{2} L /(4 E)$ and

$$
J_{C P}=\frac{1}{8} \sin \delta_{C P} \sin 2 \theta_{12} \sin 2 \theta_{13} \sin 2 \theta_{23} c_{13} .
$$

The matter effect [46-48] is included by the parameter $A$. For $\bar{\nu}_{\mu} \rightarrow \bar{\nu}_{e}$ transitions, Eq. (1) can be used by replacing $\delta_{C P} \rightarrow-\delta_{C P}$ (implying $J_{C P} \rightarrow-J_{C P}$ ) and $A \rightarrow-A$. For the two possibilities of the mass ordering, namely the normal/ inverted ordering ( $\mathrm{NO} / \mathrm{IO})$, the formula allows negative $\Delta m_{32}^{2}$, i.e. in the IO one takes negative values of $A, \alpha$ and $\Delta$. Equation (1) is derived from the series expansion in $\alpha$ where the leading order (LO), next-to-leading order (NLO), and next-to-next-to leading order (NNLO) terms correspond to the first, second plus third, and last rows of Eq. (1), respectively. ${ }^{3}$ At LO, the oscillation probability of $\nu_{\mu} \rightarrow \nu_{e}$ depends on $\theta_{13}$ and $\theta_{23}$, but is independent of $\delta_{C P}$, which appears at NLO.

Let us first focus on the $\mathrm{LO}$ and neglect the higher order terms so Eq. (1) can be approximately written as

\footnotetext{
${ }^{3}$ More exactly, the accuracy requires not only small $\alpha$, but also small $\alpha \Delta, s_{13}^{2}$, etc. See Ref. [49] for a detailed discussion on the validity of Eq. (1).
}

$$
P\left(\nu_{\mu} \rightarrow \nu_{e}\right) \approx \frac{1}{2} \sin ^{2} 2 \theta_{13}^{\mathrm{eff}} \frac{\sin ^{2}(1-A) \Delta}{(1-A)^{2}},
$$

where

$$
\sin ^{2} 2 \theta_{13}^{\mathrm{eff}}=2 s_{23}^{2} \sin ^{2} 2 \theta_{13}+\mathcal{O}(\alpha) .
$$

Here we have introduced an angle $\theta_{13}^{\text {eff }}$ which we will refer to as effective $\theta_{13}$ in this paper. At this stage it is only the coefficient $4 s_{13}^{2} c_{13}^{2} s_{23}^{2}$ of the LO expression, but below we will further generalize it to higher orders and provide a more general definition. Note that in the limit $\alpha \rightarrow 0$ and $\theta_{23} \rightarrow 45^{\circ}, \theta_{13}^{\text {eff }}$ is equal to $\theta_{13}$. At this level a correlation exists only with $\theta_{23}$ and $\theta_{13}$. For a given set of $\nu_{e}$ appearance data, if the input value of $\theta_{23}$ is increased then the output value of $\theta_{13}$ will be decreased.

Generalizing the definition of $\theta_{13}^{\text {eff }}$ by including higher order terms will be experiment-dependent since the NLO and NNLO terms have different energy dependence, which means the spectrum of the neutrino beam and the efficiency of neutrino detection have to be involved. However, by taking the approximation that the measurements are mainly determined by the total number of events, ${ }^{4}$ we can integrate out the energy dependence at NLO and NNLO, and get the following result:

$$
\begin{aligned}
\frac{1}{2} \sin ^{2} 2 \theta_{13}^{\text {eff }} \equiv & s_{23}^{2} \sin ^{2} 2 \theta_{13}-8 \alpha J_{C P} f_{s}+8 \alpha \frac{J_{C P}}{\tan \delta_{C P}} f_{c} \\
& +\alpha^{2} \sin ^{2} 2 \theta_{12} c_{23}^{2} f_{2} .
\end{aligned}
$$

Here $f_{s}, f_{c}$ and $f_{2}$ are numerical factors depending on the experimental configurations, including baseline, neutrino beam, detector, etc. For a T2K-like experiment, we have evaluated these $f$-factors, as listed in Table I. Note that the $f$-factors also depend on the mass ordering (normal/ inverted) and the oscillation channels $\left(\nu_{\mu} \rightarrow \nu_{e}\right.$ or $\left.\bar{\nu}_{\mu} \rightarrow \bar{\nu}_{e}\right)$.

Equation (5) can be used to approximately describe the correlations among the Pontecorvo-Maki-NakagawaSakata (PMNS) parameters $\theta_{13}, \theta_{23}$ and $\delta_{C P}$ with very good accuracy. In what follows we discuss some important parameter correlations based on Eq. (5). At LO, only $\theta_{23}$ and $\theta_{13}$ are correlated, which has been discussed previously. Entering the NLO, the most important observable would be $\delta_{C P}$. Here we would like to draw the reader's attention to the fact that $f_{c}$ presented here is very small ${ }^{5}$ compared to other $f$-factors. As a consequence of small $f_{c}$,

\footnotetext{
${ }^{4} \mathrm{We}$ have studied the validity of this approximation. For a T2K-like experiment, this approximation can keep valid when $N_{\text {tot }}<\mathcal{O}\left(10^{3}\right)$ or $\Delta E_{\nu} / E_{\nu}>\mathcal{O}(1 \%)$ - see more details in the appendix.

${ }^{5}$ Although this is experiment dependent, it is actually a general feature in current accelerator neutrino experiments. The reason is due to the mismatch of the cosine oscillation mode $(\cos \Delta)$ in the $C P$-even term with the sine mode in Eq. (1). A more detailed explanation requires a closer look at the shape of the neutrino flux, which is postponed to the appendix.
} 
TABLE I. The $f$ factors in T2K.

\begin{tabular}{lllcc}
\hline \hline & $\nu_{e}$ normal & $\nu_{e}$ inverted & $\bar{\nu}_{e}$ normal & $\bar{\nu}_{e}$ inverted \\
\hline$f_{s}$ & 1.42083 & -1.62128 & -1.52638 & 1.47037 \\
$f_{c}$ & 0.0314521 & -0.0159949 & 0.107811 & -0.0327648 \\
$f_{2}$ & 2.98237 & 4.04585 & 3.51664 & 3.30968 \\
\hline \hline
\end{tabular}

the $C P$-even contribution (defined by the events generated by the oscillation term proportional to $\cos \delta_{C P}$ ) is small, which makes the measurement of $\delta_{C P}$ in the experiments such as T2K and DUNE actually sensitive to $\sin \delta_{C P}$ rather than $\cos \delta_{C P}$. This explains why the uncertainties of $\delta_{C P}$ in future measurements will be maximal or minimal if the true value of $\delta_{C P}$ is $\pm 90^{\circ}$ or 0 , respectively-see, e.g., Refs. [50-53].

Next, let us look into the correlation of $\delta_{C P}$ and $\theta_{13}$. Focusing on the $\nu_{\mu} \rightarrow \nu_{e}$ mode, we draw a contour plot of $\sin ^{2} 2 \theta_{13}^{\text {eff }}\left(\theta_{13}, \delta_{C P}\right)$, as shown in Fig. 1 . Here we fix other parameters $\left(\theta_{23}, \theta_{12}\right.$ and $\left.\alpha\right)$ at their best-fit values of the global fit [6] and assume the normal ordering.

We choose five different values for $\sin ^{2} 2 \theta_{13}^{\text {eff }}$ and plot the corresponding contours. As one can see from these contours, they all appear in the shape of sine curves, which originates from the $C P$-odd term (the second term) in Eq. (5) that contains $\sin \delta_{C P}$. The $C P$-even term can be neglected because $f_{c}^{\mathrm{T} 2 \mathrm{~K}}$ is very small.

Figure 1 implies that for a fixed value of $\theta_{13}^{\text {eff }}, \theta_{13}$ approximately increases with $\sin \delta_{C P}$; it reaches the maximum (minimum) when $\delta_{C P}$ is close to $\pi / 2(-\pi / 2)$. The shape of the contours resembles the constraint on $\left(\theta_{13}, \delta_{C P}\right)$ published by the T2K collaboration (cf. Fig. 5 in Ref. [1], Fig. 39 in Ref. [4], or Fig. 9 in this paper). Indeed, given the

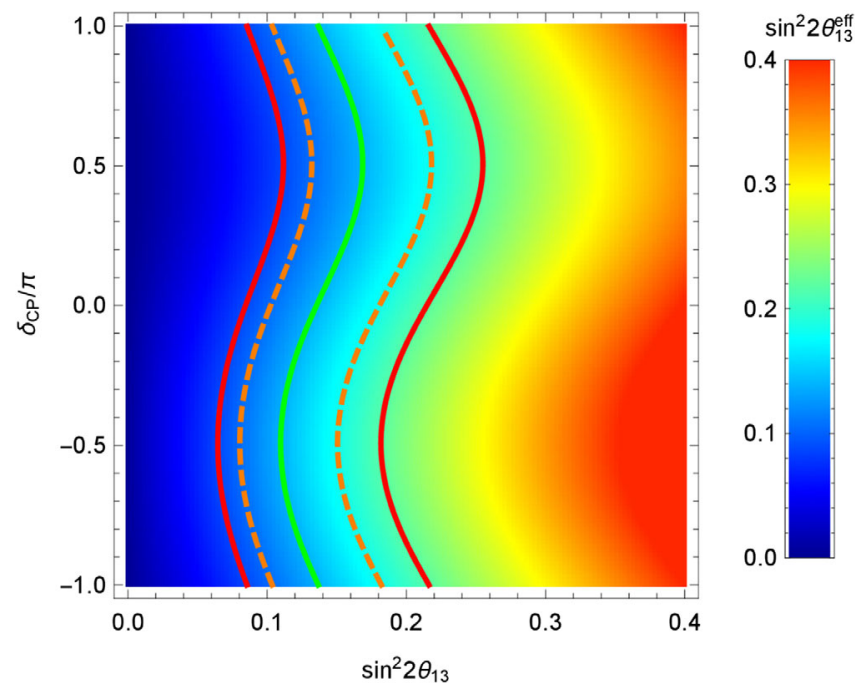

FIG. 1. Contour plot of $\sin ^{2} 2 \theta_{13}^{\text {eff }}\left(\theta_{13}, \delta_{C P}\right)$. The five contours from the left to the right correspond to $\sin ^{2} 2 \theta_{13}^{\text {eff }}=0.087,0.106$, $0.139,0.184$ and 0.218 , respectively. Normal mass ordering is assumed, with $\left(\theta_{12}, \theta_{23}, \alpha\right)=\left(34^{\circ}, 45^{\circ}, 0.031\right)$. observed numbers of events $\left(32 \nu_{e}\right.$ and $\left.4 \bar{\nu}_{e}\right)$ in $\mathrm{T} 2 \mathrm{~K}$, the constraint on $\left(\theta_{13}, \delta_{C P}\right)$ can be readily reproduced merely from a fit on $\sin ^{2} 2 \theta_{13}^{\text {eff }}$, which we present in the appendix.

Another interesting topic is about the maximal $C P$ phase $\left(\delta_{C P}=-\pi / 2\right)$ which has been the best fit value when combining the $\mathrm{T} 2 \mathrm{~K}$ result and the reactor measurements since 2013 [1]. Based on Eq. (5), we can argue analytically on when the maximal $C P$ phase would appear. Since the $C P$-even term and the $\alpha^{2}$ term are much smaller than the other terms, we neglect them in the following discussion:

$$
\frac{1}{2} S^{2} \equiv \frac{1}{2} \sin ^{2} 2 \theta_{13}^{\text {eff }} \approx s_{23}^{2} \sin ^{2} 2 \theta_{13}-8 \alpha J_{C P} f_{s} .
$$

For fixed values of $\theta_{13}, \theta_{23}$ and $\theta_{12}, S^{2}$ has a maximum $S_{\max }^{2}$ and a minimum $S_{\min }^{2}$ at $\delta_{C P}=-\pi / 2$ and $\pi / 2$, respectively. If the measured value of $S^{2}$ falls into the range of $\left(S_{\min }^{2}, S_{\max }^{2}\right)$, then one can compute the corresponding value of $\sin \delta_{C P}$ by

$$
\sin \delta_{C P}=\frac{2 \sin ^{2} 2 \theta_{13} s_{23}^{2}-S^{2}}{2 \alpha f_{s} c_{13} \sin 2 \theta_{12} \sin 2 \theta_{13} \sin 2 \theta_{23}} .
$$

If the measured $S^{2}$ is not in the range $\left(S_{\min }^{2}, S_{\max }^{2}\right)$, then there may be a tension between the $\nu_{e}\left(\bar{\nu}_{e}\right)$ appearance and disappearance data. In this case, if these data are combined, the best-fit value of $\delta_{C P}$ will be pushed to $\pm \pi / 2$. Neglecting possible tensions, from the measurement of $S^{2}$ in $\nu_{e}\left(\bar{\nu}_{e}\right)$ appearance experiments one can obtain a $C P$-dependent measurement of $\theta_{13}$, which is

$$
\begin{aligned}
\sin 2 \theta_{13} \approx & \frac{\beta \sin \delta_{C P}+\sqrt{2 s_{23}^{2} S^{2}+\beta^{2} \sin ^{2} \delta_{C P}}}{2 s_{23}^{2}}, \\
& \text { where } \beta \equiv \alpha f_{s} c_{13} \sin 2 \theta_{12} \sin 2 \theta_{23} .
\end{aligned}
$$

Although the measurement is $C P$-dependent, since $-1 \leq$ $\sin \delta_{C P} \leq 1, \theta_{13}$ measured in $\nu_{e}\left(\bar{\nu}_{e}\right)$ appearance experiments should be in the following range:

$$
\frac{\sqrt{2 s_{23}^{2} S^{2}+\beta^{2}}-|\beta|}{2 s_{23}^{2}} \lesssim \theta_{13} \lesssim \frac{\sqrt{2 s_{23}^{2} S^{2}+\beta^{2}}+|\beta|}{2 s_{23}^{2}} .
$$

Again, if the measurement of $\theta_{13}$ from reactor neutrinos is lower (or higher) than the lower (or upper) bound in Eq. (9), then the combined data fitting always prefers maximal $C P$ violating values of $\delta_{C P}$.

In summary, the effective $\theta_{13}$ analytically shows correlations of $\delta_{C P}, \theta_{13}$ and $\theta_{23}$ in the reactor and accelerator neutrino experiments. According to the dependence of $\theta_{13}^{\text {eff }}$ on $\delta_{C P}, \theta_{13}$ and $\theta_{23}$, one can understand why the $C P$-even oscillation term contributes to the $\nu_{e}$ appearance data much less than the $C P$-odd term. This implies the uncertainties of $\delta_{C P}$ measurements will be maximal or minimal if the true value of $\delta_{C P}$ is $\pm 90^{\circ}$ or 0 , respectively. Moreover, using the 
effective $\theta_{13}$, we can derive the upper and lower bounds (9) of reactor $\theta_{13}$, beyond which the combination of reactor and accelerator neutrino data should prefer maximal $C P$ violation. This can be verified in the numerical analyses in the next section.

\section{NUMERICAL RESULTS}

In this section, we adopt the numerical approach to study the phenomenologies regarding the determination and correlation of $\delta_{C P}, \theta_{13}, \theta_{23}$ and the mass ordering [54-59].

Since $\theta_{13}$ and $\theta_{23}$ can be measured independently in (anti)neutrino disappearance experiments and the measurements will be frequently used in this section, we list the recent results in Table II. The NO $\nu \mathrm{A}$ measurement [12] of $\theta_{23}$ contains two best-fit solutions. Henceforth we will refer to the two solutions in the $\theta_{23}<45^{\circ}$ and $\theta_{23}>45^{\circ}$ octants as $\mathrm{NO}_{\nu} \mathrm{A}^{-}$and $\mathrm{NO} \nu \mathrm{A}^{+}$, respectively. ${ }^{6}$ As future data will pin down the true values, it is of interest here to analyze the impact of the possible true values on the current and possible future hints of mass ordering and $C P$ phase.

For the (anti)neutrino appearance data fitting, we only focus on the T2K experiment, whose experimental parameters are more easy to access, and which has larger event numbers. Furthermore, the purpose of this paper is to note general features of parameter correlations of the parameters. For analyses including all available neutrino data, we would like to refer to the global fit work [5-7].

We use the appearance data published in [3] (taken from its Fig. 3) to construct the $\chi^{2}$-function of $\left(\theta_{23}, \theta_{13}, \delta_{C P}\right)$ while the other parameters not of interest are simply fixed at the best-fit values in the global fit. The details of event rate computation (including the cross sections, the neutrino beams) are covered by the appendix. The $\chi^{2}$-function computed from the T2K data will be referred to as $\chi_{\mathrm{T} 2 \mathrm{~K}}^{2}$ below. With this $\chi^{2}$ function, we proceed to the following phenomenological studies.

\section{A. Maximal $\boldsymbol{C P}$ violation}

As we have concluded in the analytical discussion, the appearance of maximal $C P$ violation depends on the status of the direct measurement of $\theta_{13}$. If $\theta_{13}$ measured is out of the bounds in Eq. (9), then the best fit of $\delta_{C P}$ stays at maximal values. In Fig. 2 we show the best-fit value of $\delta_{C P}$ in the $\mathrm{T} 2 \mathrm{~K} \nu_{e}\left(\bar{\nu}_{e}\right)$ appearance data, which confirms the analytical arguments. The value of $\delta_{C P}$ is computed by

$$
\min _{\delta_{C P}} \chi_{\mathrm{T} 2 \mathrm{~K}}^{2}\left(\delta_{C P}, \theta_{13}, \theta_{23}\right),
$$

where $\theta_{13}$ varies from $2^{\circ}$ to $14^{\circ}$, and $\theta_{23}$ is fixed at $46.6^{\circ}$. We compute the best-fit value of $\delta_{C P}$ for both neutrino and

\footnotetext{
${ }^{6}$ With the leading term in the muon-neutrino survival probability proportional to $\sin ^{2} 2 \theta_{23}$, solutions in both octants are naturally expected.
}

TABLE II. Recent measurements of $\theta_{13}$ and $\theta_{23}$. N and I stand for the normal and inverted mass ordering, respectively.

\begin{tabular}{|c|c|c|c|}
\hline & Daya Bay [8] & RENO [9] & Double Chooz [10] \\
\hline $\sin ^{2} 2 \theta_{13}$ & $0.0841 \pm 0.0033$ & $0.088 \pm 0.011$ & $0.111 \pm 0.018$ \\
\hline \multirow[t]{2}{*}{$\theta_{13} /{ }^{\circ}$} & $8.43_{-0.17}^{+0.17}$ & $8.62_{-0.57}^{+0.54}$ & $9.73_{-0.85}^{+0.79}$ \\
\hline & $\mathrm{T} 2 \mathrm{~K}[11]$ & $\mathrm{NO} \nu \mathrm{A}^{-}[12]$ & $\mathrm{NO}_{\nu} \mathrm{A}^{+}[12]$ \\
\hline $\sin ^{2} \theta_{23}(\mathrm{~N})$ & $0.532_{-0.068}^{+0.046}$ & $0.404_{-0.022}^{+0.030}$ & $0.624_{-0.030}^{+0.022}$ \\
\hline$\theta_{23} /{ }^{\circ}(\mathrm{N})$ & $46.8_{-3.9}^{+2.7}$ & $39.5_{-1.3}^{+1.7}$ & $52.2_{-1.8}^{+1.3}$ \\
\hline $\sin ^{2} \theta_{23}(\mathrm{I})$ & $0.534_{-0.066}^{+0.043}$ & $0.398_{-0.022}^{+0.030}$ & $0.618_{-0.030}^{+0.022}$ \\
\hline$\theta_{23} /{ }^{\circ}(\mathrm{I})$ & $46.9_{-3.8}^{+2.5}$ & $39.1_{-1.3}^{+1.8}$ & $51.8_{-1.8}^{+1.3}$ \\
\hline
\end{tabular}

antineutrino data, normal and inverted mass ordering, plotted by blue and orange, solid and dashed curves in Fig. 2 respectively.

Note that in the T2K data, the observed number of $32 \nu_{e}$ (or $4 \bar{\nu}_{e}$ ) events is larger (or smaller) than the expected number, which should be 28.7 (6.0), 24.2 (6.9), or 19.6 (7.7) for $\delta_{C P}=-\pi / 2,0$, or $\pi / 2$, respectively [60]. Since the expected number of $\nu_{e}\left(\bar{\nu}_{e}\right)$ decreases (or increases) with $\sin \delta_{C P}$, both the excess of observed $\nu_{e}$ and the deficit of $\bar{\nu}_{e}$ favor minimal $\sin \delta_{C P}$, i.e. $\delta_{C P}=-\pi / 2$.

As shown in the plot, the $\mathrm{T} 2 \mathrm{~K} \nu_{e}$ data favors a range of $\theta_{13}$ in $\left[9.2^{\circ}, 11.6^{\circ}\right]$ for the normal ordering or $\left[9.8^{\circ}, 12.7^{\circ}\right]$ for the inverted ordering. Currently reactor neutrino experiments including Daya Bay [8], RENO [9] and Double Chooz [10] all have measured smaller values of $\theta_{13}$, marked by green, magenta and black bands [ $1 \sigma$ confidence level $(\mathrm{CL})$ ] in Fig. 2. Therefore when the $\mathrm{T} 2 \mathrm{~K} \nu_{e}$ data is combined with any of the reactor measurements, the best-fit value of $\delta_{C P}$ will necessarily become about $-\pi / 2$. For the $\mathrm{T} 2 \mathrm{~K} \bar{\nu}_{e}$ data, the favored range is $\left[3.2^{\circ}\right.$, $\left.5.5^{\circ}\right]$ (normal) or $\left[2.8^{\circ}, 5.0^{\circ}\right]$ (inverted), which is smaller than the reactor measurements. So combining the T2K $\bar{\nu}_{e}$ data with any of the reactor measurements also leads to about $-\pi / 2$ for the best-fit value of $\delta_{C P}$. Note that due to

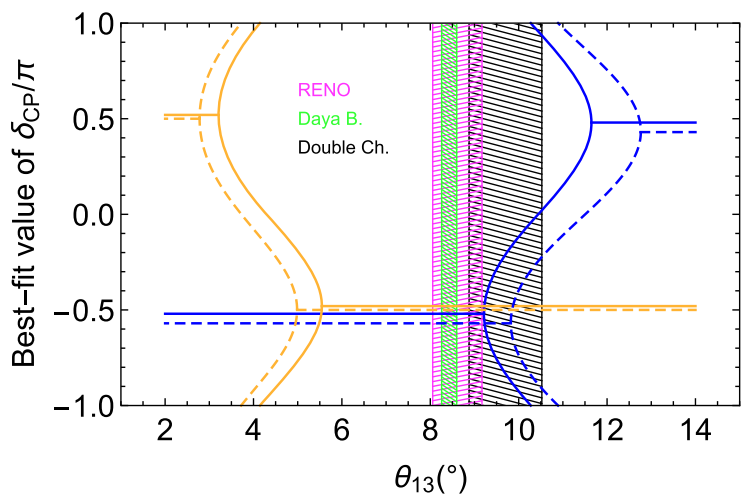

FIG. 2. The best-fit value of $\delta_{C P}$ from T2K $\nu_{e}$ (blue curve) and $\bar{\nu}_{e}$ (orange curve) data [4] as a function of $\theta_{13}$. Solid/dashed curves are for normal/inverted mass ordering. For $5.5^{\circ}<$ $\theta_{13}<9.2 r^{\circ}$, both $\nu_{e}$ and $\bar{\nu}_{e}$ data prefer a maximal $C P$ violation value $(-\pi / 2)$ of $\delta_{C P}$. 
corrections from the $C P$-even term, the actual value deviates from $-\pi / 2$ by about $\mathcal{O}(0.05 \pi)$.

\section{B. Correlation of $\boldsymbol{\theta}_{13}$ and $\delta_{C P}$}

The two-parameter fit on $\left(\theta_{13}, \delta_{C P}\right)$ will show the correlation of these two parameters. By fixing $\theta_{23}$ at certain values in $\chi_{\mathrm{T} 2 \mathrm{~K}}^{2}\left(\delta_{C P}, \theta_{13}, \theta_{23}\right)$ and allowing $\Delta \chi^{2} \leq 2.3$ in the fit, we plot the $68 \%$ CL constraints on $\left(\theta_{13}, \delta_{C P}\right)$, shown in the left panel of Fig. 3 by the blue (for $\nu_{e}$ ) and orange (for $\bar{\nu}_{e}$ ) regions. The regions within solid (dashed) lines assume normal (inverted) mass ordering.

As we can expect from Eq. (6), which shows the dependence of $\sin ^{2} 2 \theta_{13}^{\text {eff }}$ on $\sin \delta_{C P}$, the orange and blue bounds all have the shapes of sine curves. The curves of $\nu_{e}$ and $\bar{\nu}_{e}$ are bent to opposite directions: the $\nu_{e}$ curves have minimal $\theta_{13}$ at $\delta_{C P} \approx-\pi / 2$, while for $\bar{\nu}_{e}$ it is maximal. As a result, the orange region and the blue region have some overlap where $\delta_{C P}$ is mainly negative. This implies that combined fitting of $\nu_{e}$ and $\bar{\nu}_{e}$ appearance data favors negative $\delta_{C P}$. In the plots we also show the $1 \sigma$ CL bounds on $\theta_{13}$ from reactor neutrino experiments. When $\theta_{23}$ is fixed
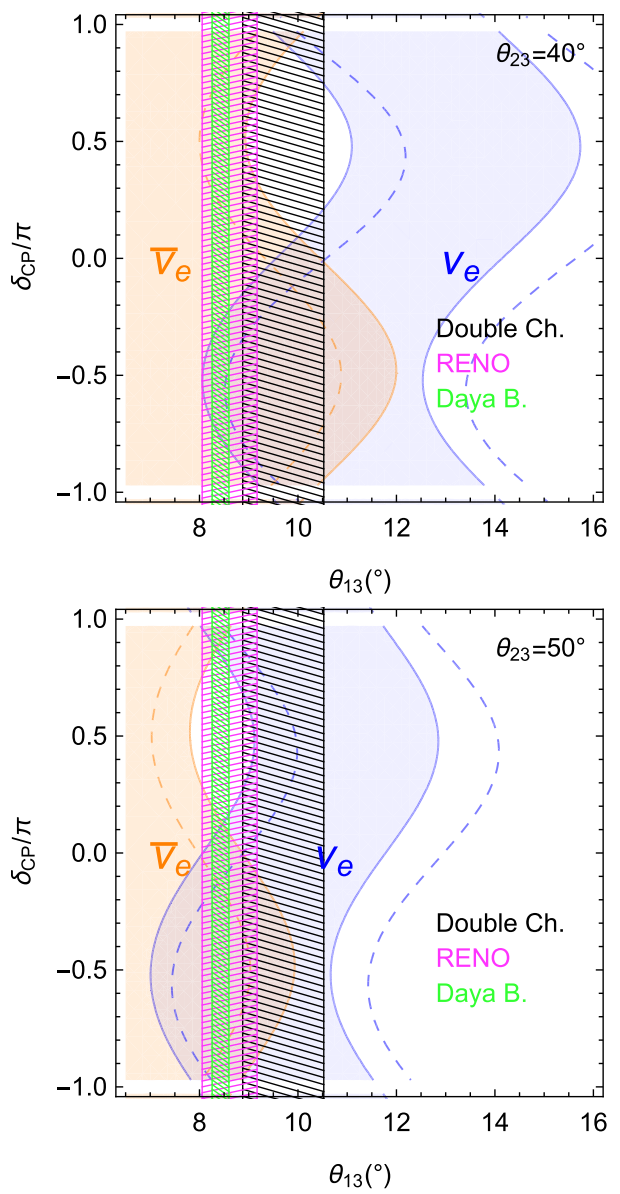

at $40^{\circ}$, the bounds from Daya Bay [8] and RENO [9] are below the overlap of the T2K $\nu_{e}$ and $\bar{\nu}_{e}$ constraints while the Double Chooz bound [10] is well compatible with the $\nu_{e}-\bar{\nu}_{e}$ overlap. However, one should notice that this depends on the value of $\theta_{23}$. If $\theta_{23}$ is fixed at the $\mathrm{NO}_{\nu} \mathrm{A}^{+}$value, as shown in the lower plots in Fig. 3, the $\nu_{e}-\bar{\nu}_{e}$ overlap covers all the reactor bounds.

In the right panel of Fig. 3, we combine the T2K data with reactor neutrino data by

$$
\begin{aligned}
\chi^{2}\left(\theta_{13}, \delta_{C P}\right)= & \chi_{\text {reactor }}^{2}\left(\theta_{13}\right)+\chi_{\mathrm{T} 2 \mathrm{~K}, \nu_{e}}^{2}\left(\theta_{13}, \delta_{C P}\right) \\
& +\chi_{\mathrm{T} 2 \mathrm{~K}, \bar{\nu}_{e}}^{2}\left(\theta_{13}, \delta_{C P}\right),
\end{aligned}
$$

where the first, second and last terms are constraints from reactor neutrino data, $\mathrm{T} 2 \mathrm{~K} \nu_{e}$ and $\bar{\nu}_{e}$ data, respectively. The $\chi^{2}$-function of $\theta_{13}$ from reactor neutrino data we adopt is

$$
\chi_{\text {reactor }}^{2}\left(\theta_{13}\right)=\left(\frac{\sin ^{2} 2 \theta_{13}-\sin ^{2} 2 \theta_{13}^{0}}{\sigma_{13}}\right)^{2}
$$
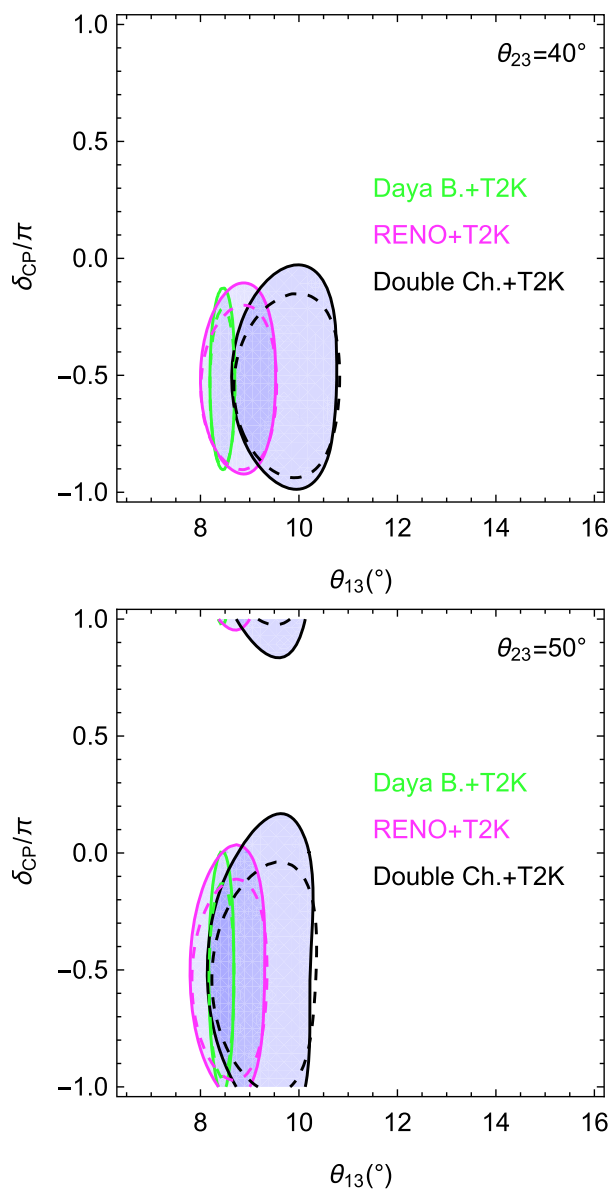

FIG. 3. Left panel: Constraints on $\left(\theta_{13}, \delta_{C P}\right)$ from T2K [4] and constraints on $\theta_{13}$ from reactor neutrino experiments. Solid/dashed curves are for normal/inverted mass ordering, blue/orange curves for $\nu_{e} / \bar{\nu}_{e}$ appearance data, respectively. Right panel: Fit results on $\left(\theta_{13}, \delta_{C P}\right)$ when $\mathrm{T} 2 \mathrm{~K}$ neutrino and antineutrino data are combined with reactor data. The upper plots apply for the $\mathrm{NO}_{\nu} \mathrm{A}^{-}$solution of $\theta_{23}$, the lower plots for $\mathrm{NO} \nu \mathrm{A}^{+}$. 
Note that Eq. (12) assumes the distribution of $\sin ^{2} 2 \theta_{13}$ to be Gaussian with a central value $\sin ^{2} 2 \theta_{13}^{0}$ and the standard deviation $\sigma_{13}$ given in Table II.

For each reactor neutrino experiment listed in Table II, we perform a $\chi^{2}$-fit combined with the $\mathrm{T} 2 \mathrm{~K}$ data and compute the corresponding 68\% CL constraints on $\left(\theta_{13}, \delta_{C P}\right)$, presented in the right panel of Fig. 3 by the green, magenta, and black contours (dashed for inverted mass ordering) for Daya Bay, RENO and Double Chooz, respectively. For all three reactor neutrino experiments the results favor negative $\delta_{C P}$. Note that the significance depends on $\theta_{23}$ : when $\theta_{23}$ increases, the bounds on $\delta_{C P}$ expand and the significance of $C P$ violation decreases. One finds from the plot that the influence of the true value of $\theta_{13}$ is not dramatic but still noteworthy; for $\theta_{23}$ in the second octant $\left(\mathrm{NO} \nu \mathrm{A}^{+}\right)$the effect is however slightly larger (note that in Fig. 3 the overlap regions of neutrino and antineutrino appearance data fits in this case better with the reactor determinations of $\theta_{13}$ ). The origin of this behavior is easy to identify in Eq. (4), where $\sin ^{2} \theta_{23}$ appears. Hence, a value of $\theta_{23}$ in the upper octant may thus reduce the current significance of the hints for maximal $C P$ violation.
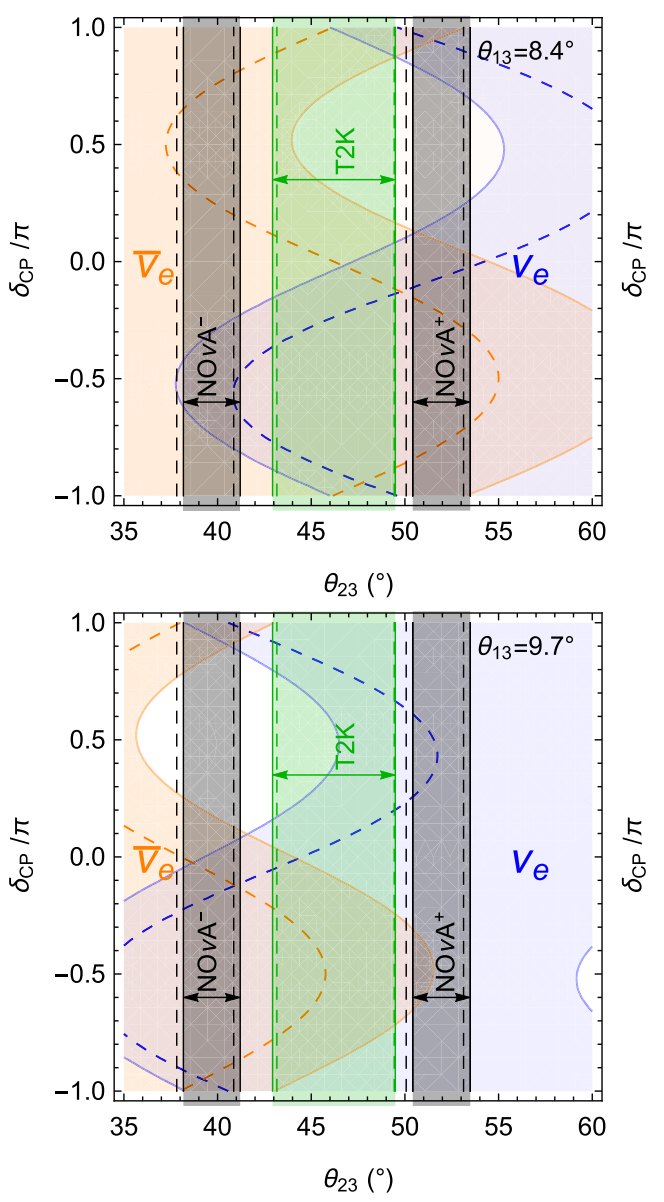

\section{Correlation of $\boldsymbol{\theta}_{23}$ and $\boldsymbol{\delta}_{C P}$}

As we have seen, $\theta_{23}$ also plays an important role in the measurement of $\delta_{C P}$ in accelerator neutrino experiments. Thus we shall study the relation of $\delta_{C P}$ and $\theta_{23}$ in more detail. Similar to Eq. (11), we have the following combined $\chi^{2}$-function:

$$
\begin{aligned}
\chi^{2}\left(\theta_{23}, \delta_{C P}\right)= & \chi_{\mathrm{Acc} \text { Dis }}^{2}\left(\theta_{23}\right)+\chi_{\mathrm{T} 2 \mathrm{~K}, \nu_{e}}^{2}\left(\theta_{23}, \delta_{C P}\right) \\
& +\chi_{\mathrm{T} 2 \mathrm{~K}, \bar{\nu}_{e}}^{2}\left(\theta_{23}, \delta_{C P}\right) .
\end{aligned}
$$

Here $\chi_{\text {Acc Dis }}^{2}$ denotes the $\chi^{2}$-function of $\theta_{23}$ constrained by accelerator neutrino disappearance experiments. In Table II, we list the measurements of $\theta_{23}$ from T2K and $\mathrm{NO} \nu \mathrm{A}$. For simplicity, in $\chi_{\text {Acc Dis }}^{2}$ we assume a Gaussian distribution of $\sin ^{2} \theta_{23}$,

$$
\chi_{\text {Acc Dis }}^{2}\left(\theta_{23}\right)=\left(\frac{\sin ^{2} \theta_{23}-\sin ^{2} \theta_{23}^{0}}{\sigma_{23}}\right)^{2},
$$

where $\sigma_{23}$ is taken as the mean value of the upper and lower uncertainties. In the left panel of Fig. 4, we show separate
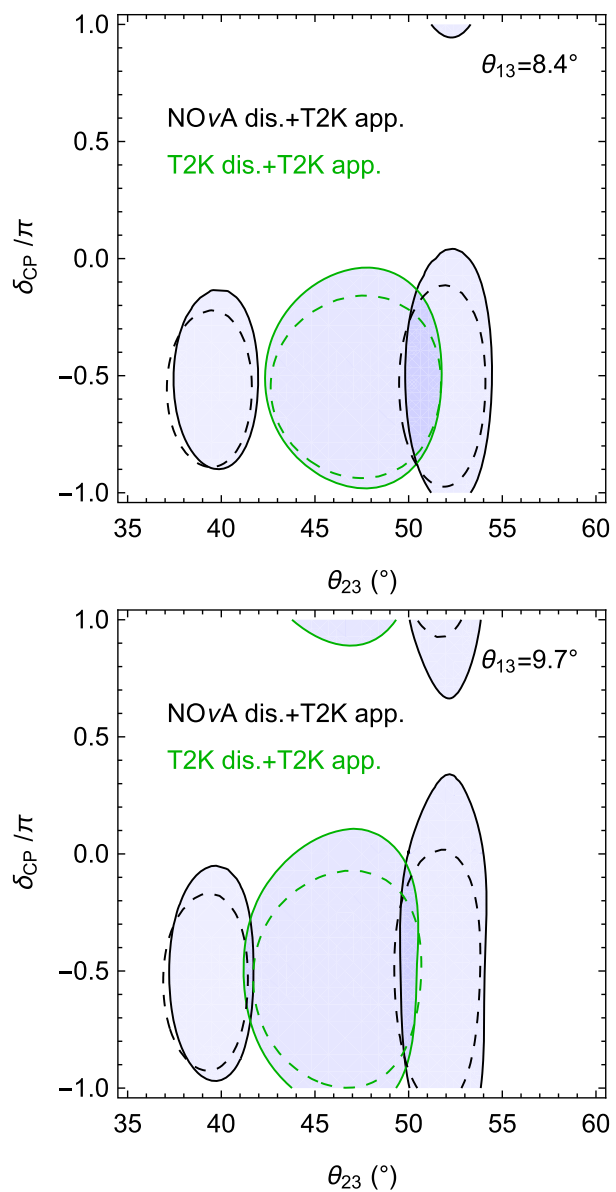

FIG. 4. Constraints on $\left(\theta_{23}, \delta_{C P}\right)$ from T2K appearance data combined with $\nu_{\mu}$ disappearance data from T2K and NO $\nu$ A. Solid/dashed curves are for normal/inverted mass ordering, blue/orange curves for $\nu_{e} / \bar{\nu}_{e}$ appearance data, respectively. The upper plots are for the best-fit value of $\theta_{13}$ from Daya Bay/RENO, the lower ones for the best-fit value of $\theta_{13}$ from Double Chooz. 
constraints on $\left(\delta_{C P}, \theta_{23}\right)$ from the three terms in Eq. (13). The constraints from the $\mathrm{T} 2 \mathrm{~K} \nu_{e}$ and $\bar{\nu}_{e}$ appearance data are presented by blue and orange regions. We can explain this behavior by rewriting Eq. (6) as

$$
s_{23}^{2} \approx \frac{S^{2}}{2 \sin ^{2} 2 \theta_{13}}+\frac{\beta}{\sin 2 \theta_{13}} \sin \delta_{C P}
$$

where $\beta$ has been defined in Eq. (8). Although $\beta$ contains $\sin 2 \theta_{23}$, for $40^{\circ}<\theta_{23}<50^{\circ}$, the dependence of $\beta$ on $\theta_{23}$ is very weak $\left(0.985 \leq \sin 2 \theta_{23} \leq 1\right)$ so one can approximately treat it as a constant with respect to $\theta_{23}$. Therefore Eq. (15) shows that the constraints on $s_{23}^{2}$ from the $\nu_{e}$ and $\bar{\nu}_{e}$ appearance data are $\delta_{C P}$ dependent and the dependence is described by a NLO correction proportional to $\sin \delta_{C P}$. Because $\beta$ in Eq. (15) has opposite signs for $\nu_{e}$ and $\bar{\nu}_{e}$, the curves are bent to opposite directions so that the blue and orange regions have overlap around $\delta=-\pi / 2$. The overlap can be approximately regarded as the region of preferred values of $\left(\delta_{C P}, \theta_{23}\right)$ by the T2K appearance data. Note that this region depends on the fixed value of $\theta_{13}$ used in Eq. (13). We choose two values $\theta_{13}=8.4^{\circ}$ and $\theta_{13}=$ $9.7^{\circ}$ (which are the central values of the Daya Bay/RENO and Double Chooz measurements, respectively) to draw the plots in Fig. 4. For both cases, $\theta_{23}$ measured from the T2K $\nu_{\mu}\left(\bar{\nu}_{\mu}\right)$ disappearance experiment (the green band) is well compatible with the appearance data. The $\mathrm{NO} \nu \mathrm{A}$ disappearance data favor nonmaximal $\theta_{23}$, showing two separate $1 \sigma$ bands (black) on the plots. For $\theta_{13}=8.4^{\circ}$, the band of $\mathrm{NO} \nu \mathrm{A}^{+}$is well compatible with the $\mathrm{T} 2 \mathrm{~K}$ appearance data

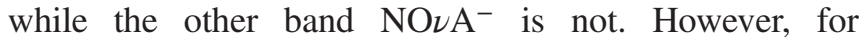
$\theta_{13}=9.7^{\circ}$, the preference moves to the band in the lower octant. In the right panel of Fig. 4 we show $1 \sigma$ constraints from a combined fit. For the combination of the T2K disappearance data with the appearance data, the $1 \sigma$ bound of $\delta_{C P}$ is $(-0.9 \pi,-0.1 \pi)$ when $\theta_{13}$ is fixed at $8.4^{\circ}$ and it expands to $(-1.1 \pi, 0.1 \pi)$ if $\theta_{13}$ is fixed at $9.7^{\circ}$. The combination of the $\mathrm{NO} \nu \mathrm{A}$ disappearance data with $\mathrm{T} 2 \mathrm{~K}$ appearance data, however, is more sensitive to the value of $\theta_{13}$. When $\theta_{13}$ is $8.4^{\circ}$, the two separate regions in black contours have $\delta_{C P}$ limited in $(-0.9 \pi,-0.1 \pi)$ or $(-1.0 \pi, 0 \pi)$. When $\theta_{13}$ is increased to $9.7^{\circ}$, both expand by about $0.1 \pi$ or $0.2 \pi$.

One finds from the plot that the influence of the true value of $\theta_{13}$ is not dramatic but nevertheless noteworthy; if $\theta_{13}$ takes the large value, the effect of $\theta_{23}$ from $\mathrm{NO} \nu \mathrm{A}^{+}$is
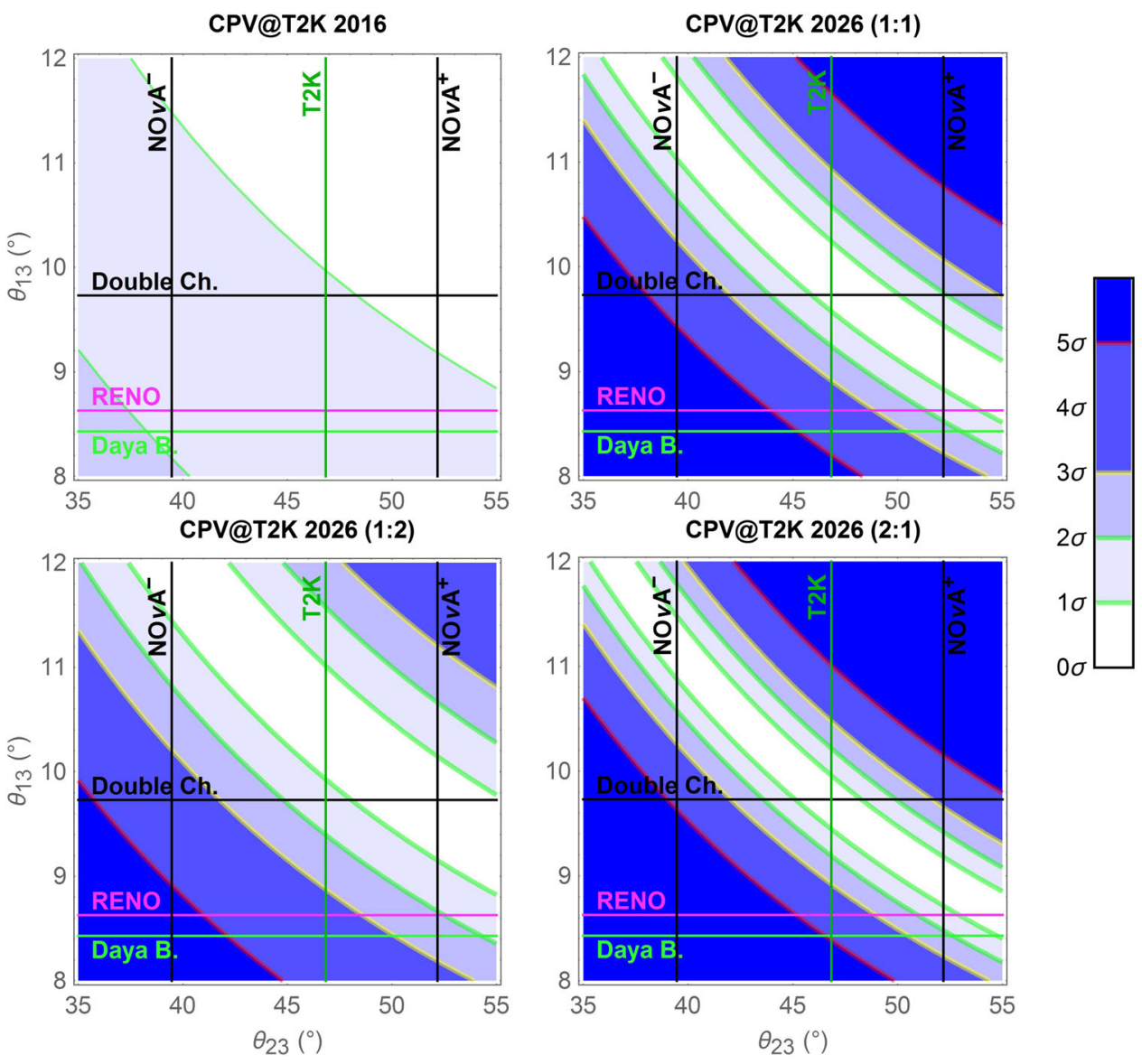

FIG. 5. $C P$ violation (CPV) in T2K data. Upper left panel: current significance of CPV in T2K (data collected until May 2016 with $1.5 \times 10^{21}$ POT [4]). Other panels: future sensitivities of CPV with $20 \times 10^{21}$ POT [63] assuming $\delta_{C P}=-\pi / 2$. The ratios [e.g. $(1: 2)$, $(2: 1)]$ denote ratios of running times in neutrino and antineutrino modes. 
TABLE III. Significance of $C P$ violation in current and future T2K. The four numbers in each bracket stand for the significance (in the unit of standard deviation $\sigma$ ) of CPV in the data of T2K2016, T2K2026 (1:1), T2K2026 (1:2) and T2K2026 (2:1), respectively, assuming that the true values of $\theta_{23}$ and $\theta_{13}$ are fixed at different best-fit values.

\begin{tabular}{lccr}
\hline \hline & $\theta_{23}=39.5^{\circ}\left({\left.\mathrm{NO} \nu \mathrm{A}^{-}\right)}\right.$ & $\theta_{23}=46.8^{\circ}(\mathrm{T} 2 \mathrm{~K})$ & $\theta_{23}=52.2^{\circ}\left(\mathrm{NO}_{2} \mathrm{~A}^{+}\right)$ \\
\hline$\theta_{13}=9.73^{\circ}$ (Double Ch.) & $(1.6,4.3,3.8,4.7)$ & $(1.1,0.5,1.4,0.1)$ & $(0.7,1.9,0.2,3.3)$ \\
$\theta_{13}=8.62^{\circ}$ (RENO) & $(1.9,6.7,5.4,7.8)$ & $(1.5,3.8,3.5,4.1)$ & $(1.3,1.7,2.1,1.4)$ \\
$\theta_{13}=8.43^{\circ}$ (Daya B.) & $(1.9,7.0,5.6,8.2)$ & $(1.6,4.4,3.9,4.8)$ & $(1.3,2.3,2.5,2.2)$ \\
\hline \hline
\end{tabular}

however slightly larger (note that in Fig. 4 the overlap regions of neutrino and antineutrino appearance data fit in

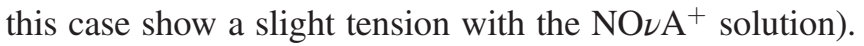
Hence, if $\theta_{23}$ lies in the second octant and $\theta_{13}$ is large (i.e. given by value of Double Chooz), then the significance of the hints for maximal $C P$ violation is reduced.

\section{Discovery of $\boldsymbol{C P}$ violation in the near future}

The current $\nu_{e}$ and $\bar{\nu}_{e}$ data of T2K, which was collected from 2010 to 2016 with $1.5 \times 10^{21}$ protons-on-target (POT), shows only a moderate hint for $C P$ Violation (CPV). Here we will investigate how this might increase with future data (see also [61,62]). The T2K experiment will keep on collecting data until 2026 and will obtain more than 10 times data $\left(20 \times 10^{21}\right.$ POT) [63], before the next generation of accelerator neutrino experiments (e.g. DUNE [44], T2HK [45] or T2HKK [64]) takes over.

To study this issue, we use the following $\chi^{2}$-function to compute the pre-DUNE significance of CPV in the data:

$$
\begin{aligned}
\chi_{\mathrm{CPV}}^{2}\left(\theta_{23}, \theta_{13}\right)= & \min _{\delta_{C P}=0 \text { or } \pi}\left[\chi_{\nu_{\mathrm{e}}+\bar{\nu}_{\mathrm{e}}}^{2}\left(\theta_{23}, \theta_{13}, \delta_{C P}\right)\right] \\
& -\min _{\delta_{C P} \in[-\pi, \pi]}\left[\chi_{\nu_{\mathrm{e}}+\bar{\nu}_{\mathrm{e}}}^{2}\left(\theta_{23}, \theta_{13}, \delta_{C P}\right)\right],
\end{aligned}
$$

where $\chi_{\nu_{\mathrm{e}}+\bar{\nu}_{\mathrm{e}}}^{2}$ is the sum of the $\chi^{2}$-functions of the $\nu_{e}$ and $\bar{\nu}_{e}$ data. Our definition $\chi_{\mathrm{CPV}}^{2}$ is the difference of how good a fit with $C P$ conservation is with respect to the $C P$ violating best-fit value. For the future data of T2K collected until 2026, we take the estimated numbers from Ref. [63] for the normal mass ordering, including $558.7 \nu_{e}$ events and 115.8 $\bar{\nu}_{e}$ events, with a background of $110.1\left(\nu_{e}\right)+63.5\left(\bar{\nu}_{e}\right)$ events. These numbers are assuming maximal $C P$ violation $\left(\delta_{C P}=-\pi / 2\right)$ and equal exposure in the neutrino and antineutrino modes, i.e. $1.0 \times 10^{22}$ POT for $\nu_{e}$ and $1.0 \times$ $10^{22}$ for $\bar{\nu}_{e}$. We will refer to this data as T2K $2026(1: 1)$. It may be favorable however to have different ratios of neutrino and antineutrino data. Hence, we also analyze the cases $(1: 2)$ and $(2: 1)$. These can be studied by rescaling the event numbers of the $(1: 1)$ mode.

The results are presented in Fig. 5, where we show the significance of CPV in both the current data (T2K 2016) and the future data (T2K 2026) with three different exposure ratios, $(1: 1),(1: 2)$ and $(2: 1)$. As we can see, compared to the T2K 2016 data, the future data will have more enhanced sensitivity on CPV. Changing the exposure ratio can affect the result. However whether this increases or decreases the significance of CPV depends on values of $\theta_{13}$ and $\theta_{23}$. For example, if $\theta_{13}=8.43^{\circ}$ and $\theta_{23}=46.8^{\circ}$, which are the best-fit values of Daya Bay and $\mathrm{T} 2 \mathrm{~K}$ disappearance measurements, then the $(2: 1)$ mode could reach $4.8 \sigma$ significance of CPV (cf. Table III), better than the $(1: 1)$ and $(1: 2)$ modes which have $4.4 \sigma$ and $3.9 \sigma$, respectively. If $\theta_{23}$ is changed to the best-fit value of $\mathrm{NO} \nu \mathrm{A}^{+}, \quad\left(\theta_{23}=52.5^{\circ}\right)$, then the $(1: 2)$ mode could reach $2.5 \sigma$, larger than $2.3 \sigma$ and $2.2 \sigma$ in the $(1: 1)$ and (2:1) modes.

At first sight it may be surprising that more neutrino data is helping the significance of $C P$ violation, rather than an equal share of more antineutrino data. The reason is that the antineutrino channel has a lower event rate (about $1 / 5$ of the neutrino channel) and a higher background (about $1: 1$ signal-to-background ratio, 4 times higher than the neutrino channel) [63].

To sum up, there is optimization potential for the $C P$ phase in future data taking. Given the large theoretical significance and interest of a maximal $C P$ phase, this could be worth exploring further. We note that since $\delta_{C P}$ is expected to receive the largest model corrections to any neutrino oscillation parameter [65], a value close to a special one like $-\pi / 2$ is a strong hint that a symmetry protects this special value, and is thus truly a worthwhile measurement.

\section{E. The mass ordering}

Finally, we would like to study the current sensitivity on the mass ordering. Here the much harder to analyze atmospheric neutrino data has large impact, ${ }^{7}$ our focus lies however just on $\nu_{e}$ and $\bar{\nu}_{e}$ appearance data. In the not so far future, ORCA [67] and PINGU [68] are also expected to contribute to the issue, and JUNO [69] will settle the question on the mass ordering with a very different method than the one based on matter effects.

We compare the normal and inverted cases by their minimal $\chi^{2}$-values,

\footnotetext{
${ }^{7}$ We note that completely independent cosmology data has, depending on used data sets, also some preference for the normal ordering; see e.g. [66].
} 


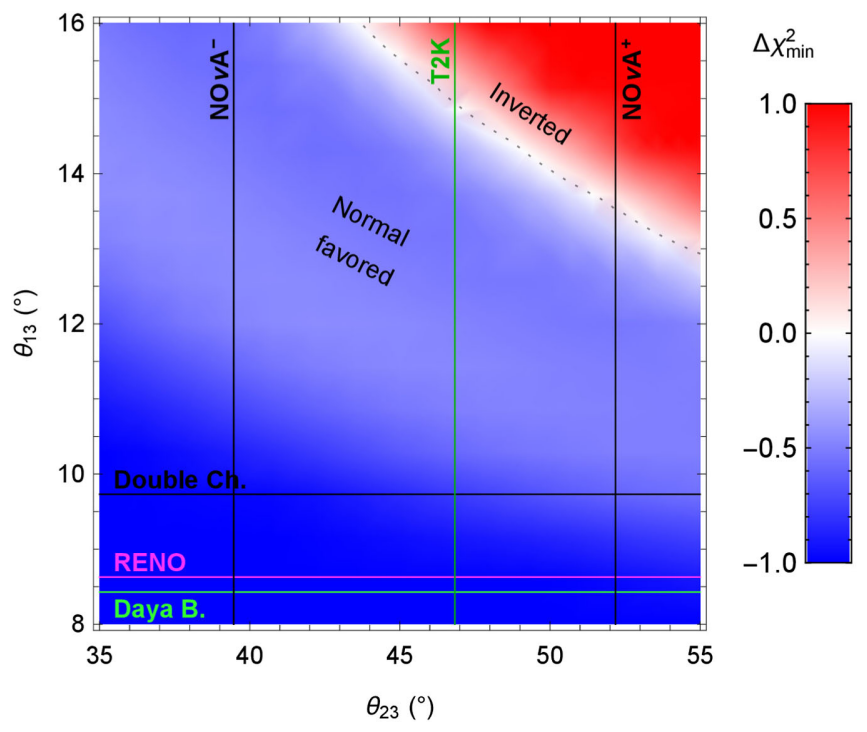

FIG. 6. Comparison of normal and inverted mass ordering by $\Delta \chi_{\min }^{2} \equiv \chi_{\operatorname{min~}}^{2}-\chi_{\min \text { I }}^{2}$ from $\mathrm{T} 2 \mathrm{~K}$ appearance data. The black dotted curve corresponds to $\Delta \chi_{\min }^{2}=0$. With the one-parameter fitting performed here, $\Delta \chi_{\min }^{2}=\sigma^{2}$.

$$
\Delta \chi_{\min }^{2} \equiv \chi_{\min \mathrm{N}}^{2}-\chi_{\operatorname{min~I}}^{2},
$$

where the subscript "N" or "I" stands for normal or inverted ordering, respectively; "min" stands for the minimization over the unknown parameter $\delta_{C P}$. More explicitly, $\chi_{\min }^{2}$ above is computed by

$\chi_{\min }^{2}\left(\theta_{23}, \theta_{13}\right)=\min _{\delta_{C P}}\left[\chi_{\nu_{\mathrm{e}}}^{2}\left(\theta_{23}, \theta_{13}, \delta_{C P}\right)+\chi_{\bar{\nu}_{\mathrm{e}}}^{2}\left(\theta_{23}, \theta_{13}, \delta_{C P}\right)\right]$,

for any given values of $\theta_{23}$ and $\theta_{13}$. Here $\chi_{\nu_{\mathrm{e}}}^{2}$ and $\chi_{\bar{\nu}_{\mathrm{e}}}^{2}$ are constraints from the $\mathrm{T} 2 \mathrm{~K} \nu_{e}$ and $\bar{\nu}_{e}$ appearance data, respectively, computed according to Table I and Table IV. The result is shown in Fig. 6, where the blue region has $\chi_{\min \mathrm{N}}^{2}<\chi_{\min \mathrm{I}}^{2}$, which means NO is favored over IO by the $\mathrm{T} 2 \mathrm{~K}$ appearance data while in the red region IO is more favored. We also show the boundary where $\Delta \chi_{\min }^{2}=0$ by the black dotted curve. Figure 6 implies that except for large $\theta_{13}$ and $\theta_{23}$, the T2K appearance data prefer NO over IO. This can also be confirmed in Fig. 3 or Fig. 4, where the overlap of the orange and blue regions becomes smaller when the mass ordering is changed from NO to IO.

\section{CONCLUSION}

Some attention has recently been cast on hints for a nontrivial $C P$ phase and the normal mass ordering. Since the combination of reactor and accelerator neutrino measurements is the origin of this we revisit the crucial parameter correlations in oscillation probabilities. Noting the different available central values of $\theta_{13}$ and $\theta_{23}$ from the different reactor and long-baseline experiments, we performed an analysis on their impact of the ranges of the
$C P$ phase and the preference of the mass ordering. While surely not a global fit including all available data, some new insights have been obtained.

To facilitate comparison of reactor and accelerator data, we have proposed the effective $\theta_{13}^{\text {eff }}$, which is defined in Eq. (5). The effective $\theta_{13}^{\text {eff }}$ can be applied to phenomenological studies of reactor and accelerator neutrino measurements. We have demonstrated that the effective $\theta_{13}^{\text {eff }}$ can be used to discuss several correlations of the neutrino parameters including $\delta_{C P}, \theta_{13}$ and $\theta_{23}$ by the T2K collaboration very well. For example, we can analytically answer the question why and when the best-fit value of $\delta_{C P}$ tends to be maximal. It can furthermore straightforwardly explain why future long-baseline experiments will be more sensitive to the $C P$ phase when $\delta_{C P}$ is around zero, rather than when it is around $-\pi / 2$. All the analytical arguments are also numerically studied and verified in this paper.

Optimization potential for the determination of $\delta_{C P}$ around the theoretically highly interesting value $-\pi / 2$ is discussed. Moreover, using the different available best-fit values of $\theta_{13}$ and $\theta_{23}$ from reactor experiments and from $\mathrm{T} 2 \mathrm{~K} / \mathrm{NO} \nu \mathrm{A}$, respectively, we studied their impact on the current ranges of $\delta_{C P}$ and the mass ordering. We have shown that, depending on the true values of $\theta_{13}$ and $\theta_{23}$, the current sensitivities are subject to change.

We hope that our study prompts some discussion on the robustness of existing hints (or any future hints) in the data, and contributes to future discussion of neutrino oscillation results.

\section{ACKNOWLEDGMENTS}

W. R. is supported by the DFG with Grant No. RO 2516/ 6-1 in the Heisenberg program.

\section{APPENDIX: THE EFFECTIVE $\theta_{13}$ APPROXIMATION}

In this appendix we derive the effective $\theta_{13}^{\text {eff }}$ including the experiment-dependent values $f_{s}, f_{c}$ and $f_{2}$; see Eq. (5). We start by writing the observable event rate in a neutrino experiment as follows:

$$
\frac{d N}{d \Omega_{f}}=\Delta t \int D\left(E_{\nu}, \Omega_{f}\right) P\left(E_{\nu}\right) \Phi\left(E_{\nu}\right) d E_{\nu} .
$$

Here $\Omega_{f}$ includes final states of all observable particles after scattering (e.g. energy/momentum of an electron), $\Delta t$ is the time of exposure, and $(\Phi, P, D)$ represent functions describing neutrino production, propagation and detection, respectively. More explicitly, $\Phi$ is the flux of neutrinos produced at the source, $P$ is the oscillation probability during propagation, and $D$ is defined as the probability of an incoming neutrino causing an event in which the final states are given by $\Omega_{f}$. Basically, $D$ is the differential cross section but practically it should also include the efficiency 
of detecting final state particles (e.g. detecting photons in photomultiplier tubes).

Integrating with respect to $\Omega_{f}$ in Eq. (A1), one can obtain the total event number. To understand the contribution of each term in Eq. (1) we decompose it into four terms:

$$
P=P_{0}+P_{s}+P_{c}+P_{2} .
$$

Here the four terms are

$$
\begin{aligned}
& P_{0}\left(E_{\nu}\right) \equiv 4 s_{13}^{2} c_{13}^{2} s_{23}^{2} p_{0}\left(E_{\nu}\right), \\
& P_{s}\left(E_{\nu}\right) \equiv-8 \alpha J_{C P} p_{s}\left(E_{\nu}\right), \\
& P_{c}\left(E_{\nu}\right) \equiv 8 \alpha J_{C P} \cot \delta_{C P} p_{c}\left(E_{\nu}\right), \\
& P_{2}\left(E_{\nu}\right) \equiv 4 \alpha^{2} s_{12}^{2} c_{12}^{2} c_{23}^{2} p_{2}\left(E_{\nu}\right),
\end{aligned}
$$

where the energy-dependent parts are defined as

$$
\begin{aligned}
& p_{0} \equiv \frac{\sin ^{2}(1-A) \Delta}{(1-A)^{2}}, \\
& p_{s} \equiv \sin \Delta \frac{\sin A \Delta}{A} \frac{\sin (1-A) \Delta}{1-A}, \\
& p_{c} \equiv \cos \Delta \frac{\sin A \Delta}{A} \frac{\sin (1-A) \Delta}{1-A}, \\
& p_{2} \equiv \frac{\sin ^{2} A \Delta}{A^{2}} .
\end{aligned}
$$

In the integral, we are only concerned about the energydependent parts of $P$ so we define

$$
\begin{aligned}
& N_{X} \equiv \Delta t \int\left(\int D d \Omega_{f}\right) p_{X} \Phi d E_{\nu}, \\
& \quad \text { for } X=0, s, c, 2 .
\end{aligned}
$$

Then the total event number will be

$$
\begin{aligned}
N_{\text {tot }}= & s_{23}^{2} \sin ^{2} 2 \theta_{13} N_{0}-8 \alpha J_{C P} N_{s}+8 \alpha \frac{J_{C P}}{\tan \delta_{C P}} N_{c} \\
& +\alpha^{2} \sin ^{2} 2 \theta_{12} c_{23}^{2} N_{2} .
\end{aligned}
$$

As we can see from Eq. (A12), by introducing $N_{0, s, c, 2}$, the dependence of the total event number on the PMNS parameters is explicitly kept. The $f$-factors introduced in the effective $\theta_{13}^{\text {eff }}$ in Eq. (5) are defined as

$$
\left(f_{s}, f_{c}, f_{2}\right) \equiv \frac{\left(N_{s}, N_{c}, N_{2}\right)}{N_{0}},
$$

which shows that they are the contributions of the different oscillation modes $p_{s}, p_{c}$ and $p_{2}$ to the total event number, in comparison to the dominant mode $p_{0}$. Since Eq. (A13) is a ratio, all overall factors (such as $\Delta t$ ) will be canceled in Eq. (A13). In practice the $f$-factors can thus be evaluated by

$$
f_{X}=\frac{\int F\left(E_{\nu}\right) p_{X}\left(E_{\nu}\right) d E_{\nu}}{\int F\left(E_{\nu}\right) p_{0}\left(E_{\nu}\right) d E_{\nu}}, \quad \text { for } X=s, c, 2,
$$

where

$$
F\left(E_{\nu}\right) \equiv\left(\int D\left(E_{\nu}, \Omega_{f}\right) d \Omega_{f}\right) \Phi\left(E_{\nu}\right) .
$$

If one disregards the efficiency of detecting final state particles (assuming it is $100 \%$ or an energy-independent constant), then the integral in Eq. (A15) can be replaced with the total cross section $\sigma\left(E_{\nu}\right)$. Therefore we have

$$
F\left(E_{\nu}\right) \propto \sigma\left(E_{\nu}\right) \Phi\left(E_{\nu}\right) .
$$

So far the discussion was general. Let us take now the $\nu_{\mu} \rightarrow \nu_{e}$ measurement of $\mathrm{T} 2 \mathrm{~K}$ as an example and evaluate the $f$-factors. The T2K experiment uses an off-axis beam of muon neutrinos generated at the J-PARC accelerator in Tokai, Japan. For $\Phi\left(E_{\nu}\right)$ in Eq. (A16), we take the $\nu_{\mu}$ flux from Ref. [70]. The Super-Kamiokande (SK) far detector (295 km away from the source) is a water Cherenkov detector in which neutrinos are detected via Cherenkov light of charged particles emitted from neutrino scattering. Since the selected $\nu_{e}$ events are expected from charged current quasielastic (CCQE) scattering, we only need the CCQE cross section for $\sigma\left(E_{\nu}\right)$ in Eq. (A16). In water $\left(\mathrm{H}_{2} \mathrm{O}\right.$, where we only consider ${ }^{16} \mathrm{O}$ ) we use the CCQE cross section data from GENIE [71]. Given the cross section and the neutrino flux, we can compute $F\left(E_{\nu}\right)$ in Eq. (A16), up to an irrelevant normalization factor. The shape of $F\left(E_{\nu}\right)$ is presented in Fig. 7.

Next we compute the integrals in Eq. (A14) with $G_{F} N_{e}=7.01 \times 10^{-14} \mathrm{eV}$ (corresponding to the terrestrial matter density $2.6 \mathrm{~g} / \mathrm{cm}^{3}$, or proton density $1.3 \mathrm{~g} / \mathrm{cm}^{3}$ ) and $\left|\Delta m_{31}^{2}\right|=2.44 \times 10^{-3} \mathrm{eV}^{2}$. The numerical results are listed in Table I, including both $\nu_{e}$ and $\bar{\nu}_{e}$ modes, NO and IO. For IO, one should use negative $\Delta m_{31}^{2}$ so $\Delta$ and $A$ are negative, leading to a negative $f_{s}$. For the $\bar{\nu}_{e}$ mode, one should change the neutrino flux and cross section correspondingly, and flip the signs of $A$ and $\delta_{C P}$, which means the negative sign before the second term of Eq. (1) should change to positive. Since we want a unified definition of $\theta_{13}^{\text {eff }}$ for both $\nu_{e}$ and $\bar{\nu}_{e}$ [i.e. the form of Eq. (A1) should also apply to $\bar{\nu}_{e}$ ], we add a negative sign in $p_{s}$ for the $\bar{\nu}_{e}$ mode. As a consequence, $f_{s}$ for $\bar{\nu}_{e}$ with NO or IO is negative or positive, respectively.

Note that $f_{c}^{\mathrm{T} 2 \mathrm{~K}}$ is much smaller than $f_{s}^{\mathrm{T} 2 \mathrm{~K}}$ which implies that at NLO the $C P$-even term in Eq. (1) has a much smaller contribution to the oscillation probability than the $C P$-odd term. Let us discuss the physical meaning of the $f$-factors 

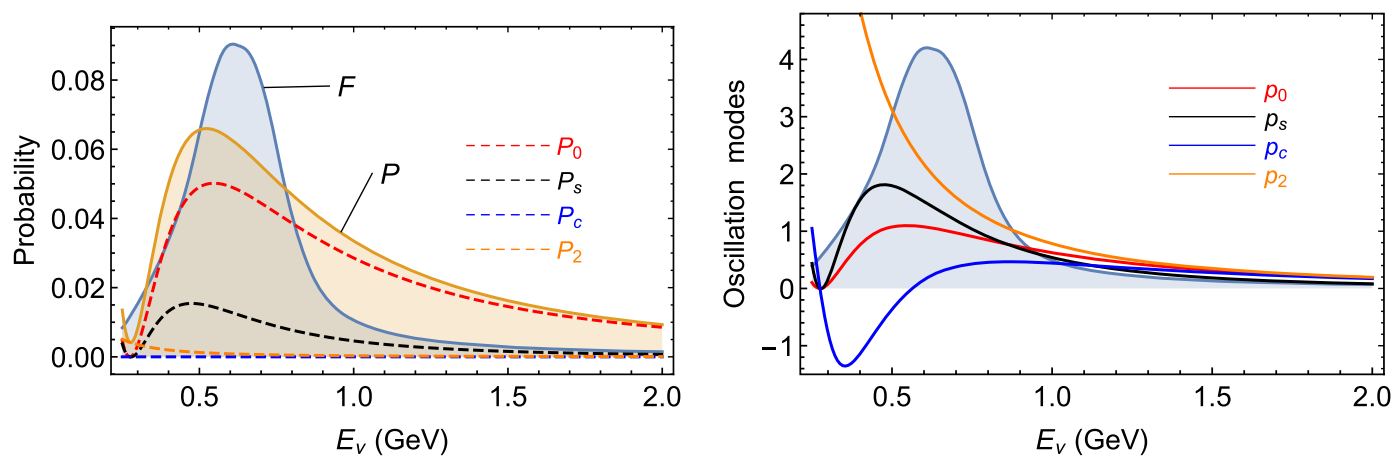

FIG. 7. The probability of $\nu_{\mu} \rightarrow \nu_{e}$ transitions and $F\left(E_{\nu}\right)$ in T2K [cf. Eqs. (A2) and (A16)]. The definitions of $P_{X}$ and $p_{X}$ $(X=0, s, c, 2)$ are given in Eqs. (A3) to (A10), $P$ is the total oscillation probability for $\left(\theta_{23}, \theta_{13}, \theta_{12}\right)=\left(45^{\circ}, 8.8^{\circ}, 34^{\circ}\right)$, $\Delta m_{13}^{2}=2.44 \times 10^{-3} \mathrm{eV}^{2}, \alpha=0.31$, and $\delta_{C P}=-\pi / 2$ (hence $P_{c}=0$ ). The function $F$ is presented in arbitrary units.

to understand why $f_{c}^{\mathrm{T} 2 \mathrm{~K}}$ is so small. As shown in Fig. 7, the function $F\left(E_{\nu}\right)$ which is proportional to both the detection rate $D$ and the flux $\Phi$, peaks at about $0.6 \mathrm{GeV}$ but is suppressed at both high and low energies. This is because if $E_{\nu}$ is too high, the flux drops exponentially; if $E_{\nu}$ is too low, the cross section suppresses $F$ (the flux drops as well). On the other hand, the oscillation probability $P$ also shows similar dependence on $E_{\nu}$ (if one only focuses on the dominating first peak of oscillation). Therefore, in order to obtain sizable event numbers, it is important to make $F$ (which is essentially flux times cross section) and the probability $P$ overlap as much as possible when they obtain their maximal values.

In the left panel of Fig. 7, we show the contribution of each term of the probability, i.e. $P_{0}, P_{s}, P_{c}$ and $P_{2}$ [cf. Eqs. (A2)-(A6)]. In the right panel, we focus on the pure oscillation modes, $p_{0}, p_{s}, p_{c}$ and $p_{2}$ [cf. Eqs. (A7)(A10)] which are directly related to the $f$-factors according to Eq. (A14). The physical meaning of $f_{X}(X=s, c, 2)$ is how much the corresponding oscillation mode $p_{X}$ and the function $F\left(E_{\nu}\right)$ overlap, which determines the sensitivity of the experiment on this mode itself. As we can see, $p_{c}$ has the least overlap with the shaded region corresponding to $F$, and it also contains a negative part which causes cancellation with the positive part. This explains why $f_{c}$ is much smaller than the other $f$-factors.

Although the smallness of $f_{c}$ is experiment dependent, it should be a general feature in accelerator neutrino experiments because usually the experiments should be designed so that $F\left(E_{\nu}\right)$ peaks at the same energy where the oscillation mode $\sin ^{2} \Delta$ peaks. The oscillating part in $p_{c}$ [cf. (A9)] approximates in the valid limit of small matter effects as $\sin \Delta \cos \Delta$, which if integrated with the function $F$ (see Fig. 7) will always lead to the similar cancellation. Therefore one can conclude that in general $f_{c}$ is small in such experiments.

Finally, we would like to discuss the validity of the effective $\theta_{13}^{\text {eff }}$ when applied to accelerator neutrino experiments. When introducing the effective $\theta_{13}^{\text {eff }}$, we actually assume that the observable effects of the NLO and NNLO terms are only their contributions to the total event number; i.e. the distortion of the shape of $\nu_{e}\left(\bar{\nu}_{e}\right)$ distribution due to the different oscillation modes is assumed to be negligible. However, for experiments with very high sensitivities, this assumption might not hold. To understand the differences between the effective $\theta_{13}$ and the conventional treatment, we compare the event rates in Fig. 8. The event rates are plotted in arbitrary units, computed via $F\left(E_{\nu}\right) \times P\left(E_{\nu}\right)$ where $P\left(E_{\nu}\right)$ takes either the form of Eq. (3) [with $\theta_{13}^{\text {eff }}$ defined in (5)] or of Eq. (1), plotted by the blue dashed or the black solid curve, respectively. From Fig. 8 we can see that the difference between the two curves is very small, which implies the approximation adopted in the effective $\theta_{13}$ could make a difference only in experiments with very high event numbers (i.e. very small statistical uncertainties) as well as correspondingly very small systematic uncertainties. More explicitly, if we assume the difference is about $2 \%$ as a typical value indicated in Fig. 8, then the total event number $N_{\text {tot }}$ should be (according to

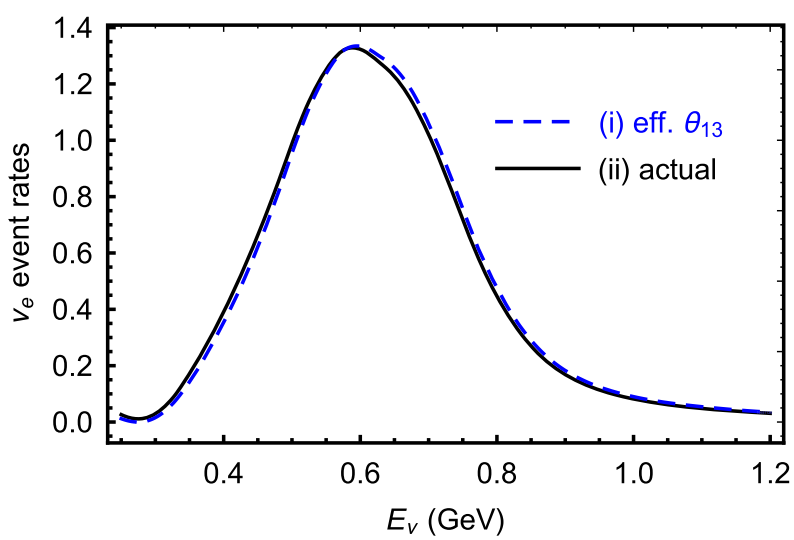

FIG. 8. Comparison of $\nu_{e}$ event rates (in arbitrary units) computed (i) from the effective $\theta_{13}^{\text {eff }}$ formula [cf. Eqs. (3) and (5)] and (ii) from the actual oscillation formula [cf. Eq. (1)] applied to $\mathrm{T} 2 \mathrm{~K}$. 


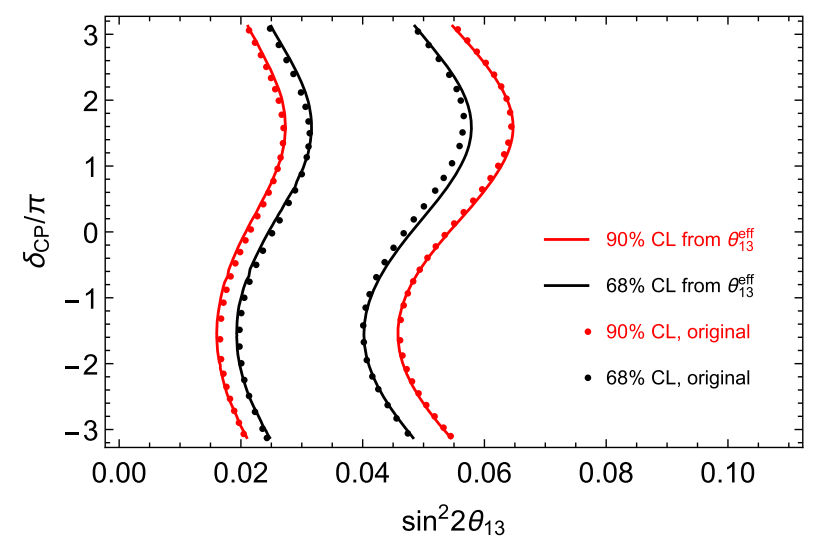

FIG. 9. Reproduced constraints (red and black curves) on $\left(\theta_{13}, \delta_{C P}\right)$ from our effective $\theta_{13}$ framework compared to the original $\mathrm{T} 2 \mathrm{~K}$ result [4] (red and black points) for the normal mass ordering. The agreement shows that the effective $\theta_{13}$ framework is very accurate to describe the sensitivity of $\mathrm{T} 2 \mathrm{~K}$ $\nu_{\mu} \rightarrow \nu_{e}$ data on $\left(\theta_{13}, \delta_{C P}\right)$.

$1 / \sqrt{N_{\text {tot }}} \sim 2 \%$ ) larger than $\mathcal{O}\left(10^{3}\right)$ to distinguish between the two curves. Current on-going accelerator neutrino experiments such as T2K, MINOS, NO $\nu \mathrm{A}$ cannot reach such high statistics while the future DUNE experiment will do so $\left(861 \nu_{e}\right.$ events and $167 \bar{\nu}_{e}$ events for 4 years of running [44]). However, the above requirement does not take the energy resolution into account (i.e. assumes no uncertainty in the energy measurement). Since the areas under the two curves are the same (by definition), the energy resolution has to be very high to see any differences between the two curves. The horizontal differences are typically around $\mathcal{O}(1 \%)$, which implies that the energy resolution should reach $\Delta E_{\nu} / E_{\nu}<\mathcal{O}(1 \%)$ to distinguish between them. Therefore, we can draw the conclusion that the approximation in the effective $\theta_{13}$ should be valid for experiments with $N_{\text {tot }}<\mathcal{O}\left(10^{3}\right)$ or $\Delta E_{\nu} / E_{\nu}>\mathcal{O}(1 \%)$.

Using the effective $\theta_{13}$ framework, we can approximately reproduce the constraint of the $\mathrm{T} 2 \mathrm{~K}$ data on $\left(\theta_{13}, \delta_{C P}\right)$, as shown in Fig. 9. Compared with the original constraint (black and red points) from Ref. [4], our reproduced result agrees with it very well. This implies that our effective $\theta_{13}$ framework is able to quantitatively describe the sensitivity of T2K $\nu_{\mu} \rightarrow \nu_{e}$ data on $\left(\theta_{13}, \delta_{C P}\right)$
TABLE IV. The $k$ and $b$ parameters for the T2K data.

\begin{tabular}{ccccc}
\hline \hline & $\nu_{e}$ normal & $\nu_{e}$ inverted & $\bar{\nu}_{e}$ normal & $\bar{\nu}_{e}$ inverted \\
\hline$n$ & 32 & 32 & 4 & 4 \\
$k$ & $1.86 \times 10^{2}$ & $1.55 \times 10^{2}$ & 51.5 & 55.9 \\
$b$ & 4.8 & 5.4 & 2.5 & 2.5 \\
\hline \hline
\end{tabular}

with sufficient accuracy. The details of reproducing the constraint are given next.

Since the expected event number $\mu$ should linearly depend on the probability of $\nu_{e}$ appearance, it should also linearly depend on $S^{2} \equiv \sin ^{2} 2 \theta_{13}^{\text {eff }}$. So we can write

$$
\mu=k S^{2}+b
$$

where $k$ is a scale factor and $b$ can be regarded as the background because it equals the expected event number without neutrino oscillation. The observed event number $n$ should obey a Poisson distribution; hence the $\chi^{2}$-function of $\mu$ is

$$
\chi^{2}(\mu)=2\left(\mu-n+n \ln \frac{n}{\mu}\right)
$$

By plugging Eq. (A17) into Eq. (A18) we obtain the $\chi^{2}$-function of $S^{2}$,

$$
\chi^{2}\left(S^{2}\right)=2\left(k S^{2}+b-n+n \ln \frac{n}{k S^{2}+b}\right) .
$$

In the recent $\mathrm{T} 2 \mathrm{~K}$ appearance data, the observed event number $n$ is $32\left(\nu_{e}\right)$ or $4\left(\bar{\nu}_{e}\right)$; the two parameters $k$ and $b$ can be determined by comparing Eq. (A19) to the original T2K result [4], which are listed in Table IV.

Equation (A19) can be further converted to the $\chi^{2}$-function of $\left(\theta_{13}, \delta_{C P}\right)$ if $\alpha, \theta_{23}$ and $\theta_{12}$ are fixed or marginalized over. In Fig. 9 we simply use fixed values $\alpha=0.0307, \theta_{23}=46.5^{\circ}$ and $\theta_{12}=33.4^{\circ}$ to generate the black and red curves. Although the original T2K result was obtained by marginalizing all nuisance parameters, our result using fixed values already agrees well with it.
[1] K. Abe et al. (T2K Collaboration), Phys. Rev. Lett. 112, 061802 (2014).

[2] J. Bian et al. ( $\mathrm{NO} \nu \mathrm{A}$ Collaboration), in Proceedings, Meeting of the APS Division of Particles and Fields (DPF 2015): Ann Arbor, Michigan, USA, 4-8 August 2015 (2015).
[3] K. Abe et al. (T2K Collaboration), Phys. Rev. Lett. 118, 151801 (2017).

[4] K. Abe et al. (T2K Collaboration), Phys. Rev. D 96, 092006 (2017).

[5] F. Capozzi, E. Lisi, A. Marrone, D. Montanino, and A. Palazzo, Nucl. Phys. B908, 218 (2016). 
[6] I. Esteban, M. C. Gonzalez-Garcia, M. Maltoni, I. MartinezSoler, and T. Schwetz, J. High Energy Phys. 01 (2017) 087.

[7] P. F. de Salas, D. V. Forero, C. A. Ternes, M. Tortola, and J. W. F. Valle, arXiv:1708.01186.

[8] F. P. An et al. (Daya Bay Collaboration), Phys. Rev. D 95, 072006 (2017).

[9] H. Seo et al. (RENO Collaboration), J. Phys. Conf. Ser. 718, 062053 (2016).

[10] T. Matsubara et al. (Double Chooz Collaboration), Proc. Sci., ICHEP2016 (2016) 469.

[11] K. Abe et al. (T2K Collaboration), Phys. Rev. D 96, 011102 (2017).

[12] P. Adamson et al. (NO $\nu$ A Collaboration), Phys. Rev. Lett. 118, 151802 (2017).

[13] Http://nova-docdb.fnal.gov/cgi-bin/RetrieveFile?docid=25938 \&filename=radovicJETPFinalPublic.pdf \&version $=1$.

[14] P. F. Harrison and W. G. Scott, Phys. Lett. B 547, 219 (2002).

[15] E. Ma, Phys. Rev. D 66, 117301 (2002).

[16] K. Babu, E. Ma, and J. Valle, Phys. Lett. B 552, 207 (2003).

[17] E. Ma, Mod. Phys. Lett. A 17, 2361 (2002).

[18] W. Grimus and L. Lavoura, Phys. Lett. B 579, 113 (2004).

[19] C. C. Nishi, Phys. Rev. D 88, 033010 (2013).

[20] E. Ma, Phys. Rev. Lett. 112, 091801 (2014).

[21] S. Fraser, E. Ma, and O. Popov, Phys. Lett. B 737, 280 (2014).

[22] G.-N. Li and X.-G. He, Phys. Lett. B 750, 620 (2015).

[23] E. Ma, A. Natale, and O. Popov, Phys. Lett. B 746, 114 (2015).

[24] A. Di Iura, C. Hagedorn, and D. Meloni, J. High Energy Phys. 08 (2015) 037.

[25] R. N. Mohapatra and C. C. Nishi, J. High Energy Phys. 08 (2015) 092.

[26] Y.-L. Zhou, arXiv:1409.8600.

[27] A. S. Joshipura and K. M. Patel, Phys. Lett. B 749, 159 (2015).

[28] H.-J. He, W. Rodejohann, and X.-J. Xu, Phys. Lett. B 751, 586 (2015).

[29] H.-J. He and X.-J. Xu, Phys. Rev. D 86, 111301 (2012).

[30] W. Rodejohann and X.-J. Xu, Phys. Rev. D 96, 055039 (2017).

[31] W. Rodejohann and X.-J. Xu, Eur. Phys. J. C 76, 138 (2016).

[32] Z.-h. Zhao, J. High Energy Phys. 09 (2017) 023.

[33] C. C. Nishi and B. L. Sanchez-Vega, J. High Energy Phys. 01 (2017) 068.

[34] P. Chen, G.-J. Ding, F. Gonzalez-Canales, and J. W. F. Valle, Phys. Lett. B 753, 644 (2016).

[35] T. Fukuyama, Prog. Theor. Exp. Phys. 2017, $033 \mathrm{~B} 11$ (2017).

[36] C.-C. Li and G.-J. Ding, J. High Energy Phys. 05 (2015) 100.

[37] M. Freund, Phys. Rev. D 64, 053003 (2001).

[38] A. Cervera, A. Donini, M. B. Gavela, J. J. Gomez Cádenas, P. Hernández, O. Mena, and S. Rigolin, Nucl. Phys. B579, 17 (2000).

[39] K. Olive et al. (Particle Data Group), Chin. Phys. C 38, 090001 (2014).
[40] K. Abe et al. (T2K Collaboration), Phys. Rev. Lett. 111, 211803 (2013).

[41] P. Adamson et al. (MINOS Collaboration), Phys. Rev. Lett. 106, 181801 (2011).

[42] D. Ayres et al. (NO $\nu$ A Collaboration), arXiv:hep-ex/ 0503053.

[43] R. Patterson (NO $\nu$ A Collaboration), Nucl. Phys. B, Proc. Suppl. 235-236, 151 (2013).

[44] R. Acciarri et al. (DUNE Collaboration), arXiv:1512.06148.

[45] K. Abe et al. (Hyper-Kamiokande Working Group), arXiv: 1412.4673 .

[46] L. Wolfenstein, Phys. Rev. D 17, 2369 (1978).

[47] S. Mikheev and A. Y. Smirnov, Sov. J. Nucl. Phys. 42, 913 (1985).

[48] S. Mikheev and A. Y. Smirnov, Nuovo Cimento Soc. Ital. Fis. 9C, 17 (1986).

[49] X.-J. Xu, J. High Energy Phys. 10 (2015) 090.

[50] N. Nath, M. Ghosh, and S. Goswami, Nucl. Phys. B913, 381 (2016).

[51] P. Ballett, S. F. King, S. Pascoli, N. W. Prouse, and T. Wang, Phys. Rev. D 96, 033003 (2017).

[52] S. Fukasawa, M. Ghosh, and O. Yasuda, Nucl. Phys. B918, 337 (2017).

[53] P. Coloma, A. Donini, E. Fernandez-Martinez, and P. Hernandez, J. High Energy Phys. 06 (2012) 073.

[54] H. Minakata, H. Sugiyama, O. Yasuda, K. Inoue, and F. Suekane, Phys. Rev. D 68, 033017 (2003); 70, 059901E (2004).

[55] P. Huber, M. Lindner, T. Schwetz, and W. Winter, Nucl. Phys. B665, 487 (2003).

[56] H. Minakata and H. Sugiyama, Phys. Lett. B 580, 216 (2004).

[57] O. Mena and S. J. Parke, Phys. Rev. D 70, 093011 (2004).

[58] A. Ghosh, T. Thakore, and S. Choubey, J. High Energy Phys. 04 (2013) 009.

[59] S. Choubey and A. Ghosh, J. High Energy Phys. 11 (2013) 166.

[60] L. Magaletti et al. (T2K Collaboration), Proc. Sci., NOW2016 (2017) 003.

[61] M. Ghosh, S. Goswami, and S. K. Raut, Mod. Phys. Lett. A 32, 1750034 (2017).

[62] M. Ghosh, Phys. Rev. D 93, 073003 (2016).

[63] K. Abe et al., arXiv:1609.04111.

[64] K. Abe et al. (Hyper-Kamiokande proto-Collaboration), arXiv:1611.06118.

[65] W. Rodejohann and X.-J. Xu, Nucl. Phys. B899, 463 (2015).

[66] S. Hannestad and T. Schwetz, J. Cosmol. Astropart. Phys. 11 (2016) 035.

[67] S. Adrian-Martinez et al. (KM3Net Collaboration), J. Phys. G 43, 084001 (2016).

[68] M. G. Aartsen et al. (IceCube PINGU Collaboration), arXiv:1401.2046.

[69] F. An et al. (JUNO Collaboration), J. Phys. G 43, 030401 (2016).

[70] K. Abe et al. (T2K Collaboration), Phys. Rev. D 88, 032002 (2013).

[71] C. Andreopoulos et al., Nucl. Instrum. Methods Phys. Res., Sect. A 614, 87 (2010). 